\title{
Workable Monitoring System based on Spaceborne SAR Images for Mining Areas - STINGS Development Project
}

\author{
C. H. Yang ${ }^{1, *}$, A. Müterthies ${ }^{1}$, U. Soergel ${ }^{2}$ \\ ${ }^{1}$ EFTAS Remote Sensing Transfer of Technology, Münster, Germany - (chia-hsiang.yang, andreas.mueterthies)@eftas.com \\ ${ }^{2}$ Institute for Photogrammetry, University of Stuttgart, Stuttgart, Germany - soergel@ifp.uni-stuttgart.de
}

KEY WORDS: Differential Interferometric Synthetic Aperture Radar (DInSAR), Persistent Scatterer Interferometry (PSI), Small Baseline Subset (SBAS), Mining Monitoring, Sentinel-1, Disaster Prevention

\begin{abstract}
:
Nowadays monitoring of mining areas, e.g., regarding dam stability, has become increasingly important with rising awareness of safety and environmental protection. An appropriate monitoring scheme is necessitated to legally activate, reactivate, or terminate mining operations. Usually such monitoring relies on in situ surveys, which are unrealistic to cover an extensive mining area. Alternatively, remote sensing based on spaceborne data offers efficient and cost-effective solutions for regular surveillance of large areas. Spaceborne SAR sensors provide images captured rapidly over vast areas at fine spatiotemporal resolution. These sensors are characterized by weather independent and day-and-night vision, which guarantees intensive image series without cloud occlusion. Using multi-temporal SAR images, advanced DInSAR such as PSI and SBAS is a mature technique to evaluate surface deformation at best millimetre level. This technique has been commercialized as a standard service in many Geoinformation companies. Nevertheless, experts from other fields like mining engineers often doubt the information about movement derived from DInSAR. Our duty in industry is to solve these doubts and tailor our techniques for various applications. With the support of STINGS project, we have developed an initial prototype of our monitoring system. The final goal is to launch an interactive GIS-based platform as an early warning system to the public. In this paper, we demonstrate our initial test result using Sentinel-1 images at a mining site in Chile. We also propose the strategies to solve the problems in real applications and discuss how to improve the overall quality.
\end{abstract}

\section{INTRODUCTION}

For many countries, mining industry accounts for an important share of their economic outputs. In general, open-pit and underground mining activities cause structural deformation and ground subsidence. Monitoring such a mining impact has become increasingly important for safety of human lives and properties. Recently, two catastrophic dam collapses (https://en.wikipedia. org/wiki/Mariana_dam_disaster \& https://en.wikipedia.org/wiki /Brumadinho_dam disaster) in Brazil causes miserable loss of human lives (205 deaths and 122 missing combined) and countless properties in 2015 and 2019. The post investigation of the first event indicates there were signs of structural damages on the dam reported from on-site measurement. We believe such a disaster could be prevented with a proper monitoring and earlywarning system.

Normally, monitoring routines rely on in situ surveys, which are considered reliable and accurate under proper control while expensive and time-consuming. A regular measurement campaign over an extensive area is not economically effective. Alternatively, remote sensing based on spaceborne data offers an efficient and cost-effective way. This technique aims at frequent surveillance of large areas, which provides comparable complements to in situ measurement data. The information derived from both data sources must be integrated to establish a workable monitoring system.

Spaceborne SAR sensors deliver radar images, which are acquired regularly over vast areas at fine spatiotemporal resolution. For example, TerraSAR-X operating in HighResolution Spotlight Mode delivers a new duplicate image every 11 days, which covers a specific area of $5 \mathrm{~km} \times 5 \mathrm{~km}$ with around
$1 \mathrm{~m}$ resolution. Such image series are further used in interferometry techniques for scene monitoring. Moreover, active SAR sensors are weather independent and have a day-andnight vision ability. This advantage makes SAR images free of cloud occlusion and always available for use. These characteristics make SAR suitable for long-term monitoring tasks.

Differential interferometric SAR (DInSAR) using only two SAR images evaluates surface movement commonly up to centimetre level. This technique has been refined in persistent scatterer interferometry (PSI) (Crosetto et al., 2016; Ferretti et al., 2000, 2001, 2011; Hooper et al., 2004; Kampes, 2006) and small baseline subset (SBAS) (Berardino et al., 2002; Lanari et al., 2007). Basically, both approaches look for strong, stable, and coherent radar signals, as measurement points, from a SAR image sequence. Certain point properties like temporal coherence, line-of-sight (LOS) velocity ( $\mathrm{mm} /$ year level), topography height, geographic position, etc. are derived for scene monitoring.

Many Geoinformation companies offer interferometry monitoring as a standard service. Following launches of Sentinel1 satellites, costs of such monitoring works are dramatically reduced and affordable to many needs of different fields in the market. Nevertheless, there are still limitations and difficulties when applying interferometry to real world. For example, how to separate and extract building subsidence from other components caused by variation of temperature and soil moisture. In addition, many experts and customers have doubts about interferometry results. How can a sensor in space be capable of detecting so small deformation on earth? Can we trust the movements evaluated from SAR signals or not?

\footnotetext{
* Corresponding author
} 
In STINGS project (https://eitrawmaterials.eu/project/stings/), we have developed an initial prototype of our monitoring system for mining areas. We first focus on monitoring of dam and tailing. The system can be later converted for other applications, e.g., monitoring of underground pipelines or ground subsidence caused by past mining campaigns. The main objective is to solve the real problems and improve the current interferometry tools in practice. We aim to launch an interactive GIS-based platform as an early warning system in the market.

In this paper, we first introduce our monitoring system in Section 2. Section 3 illustrates the initial test at the mining site in Chile. We also describe the strategies in Section 4 to solve the problems and questions that are often faced in practice. Some examples are also discussed here. The conclusions are finally summarized in Section 5 .

\section{MONITORING SYSTEM}

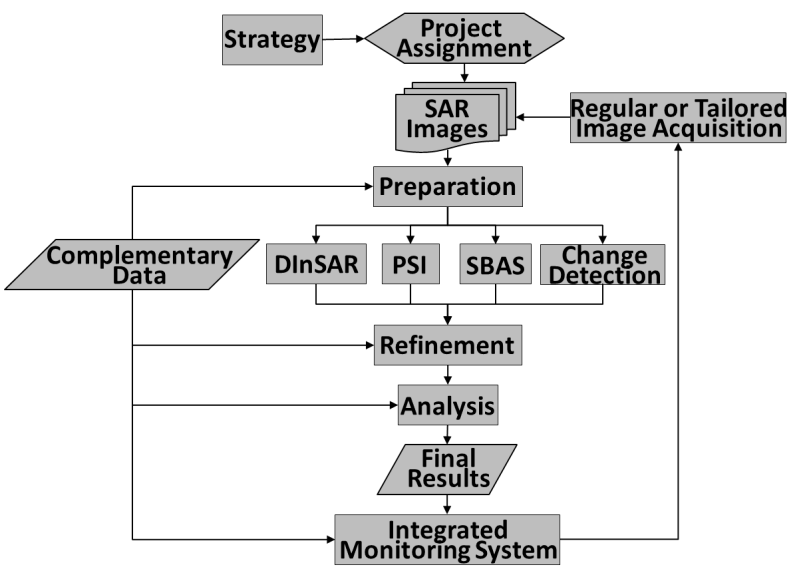

Figure 1. Monitoring system

The flowchart of our monitoring system is illustrated in Figure 1. Here we introduce the overall concept and detail the context in the following subsections. First of all, we look into the project protocol to assign the tasks along with strategies. The objectives and requirements must be clarified and confirmed. Given a dam surveillance for safety, people should decide precision, accuracy, resolution, repetition, data format, risk indexing, etc. These decisions have big influences on the subsequent works. We choose adequate image sources and acquire images suitable to projects. The preparation phase prepares for interferometry processing, including image pre-processing, cutting of interest areas, parameter definition, evaluation of time requirement, machine assignment, and so on. Afterwards, DInSAR, PSI, SBAS, or change detection are performed depending on project needs. The initial results are first refined to remove noise (unwanted signals), extract components of interest, improve overall quality, etc. The refined results are then analysed to evaluate their precision and accuracy. These two post-processing steps are developed based on data-driven statistics. Moreover, we also involve complementary data, if any, which are derived from other measurements and ground truth. The final results would be wrapped as vector files like shapefile. Each measurement point contains certain attributes such as ID, geographic coordinate, height, deformation velocity, displacement series, precision, accuracy, risk index, etc. Finally, the vector data are integrated into an interactive GIS-based platform, which not only provides real-time and 24/7 information but also operates as an earlywarning system.

\subsection{Spaceborne SAR images}

Commercial spaceborne SAR sensors (Table 1) are currently in operation with X, C, and L bands. Three typical examples are TerraSAR-X, Sentinel-1, and PALSAR-2, respectively. Other operational and planned satellites include PAZ (X), COSMOSkyMed (X), Radarsat-2 (C), RCM (C), SAOCOM (L), NISAR (L), and TanDEM-L (L).

\begin{tabular}{|c|c|c|c|}
\hline Wavelength & $\begin{array}{c}\text { X band } \\
(\sim 3.1 \mathrm{~cm})\end{array}$ & $\begin{array}{c}\text { C band } \\
(\sim 5.6 \mathrm{~cm})\end{array}$ & $\begin{array}{c}\text { L band } \\
(\sim 22.9 \mathrm{~cm})\end{array}$ \\
\hline Satellite & TerraSAR-X & Sentinel-1 & PALSAR-2 \\
\hline Resolution & $0.24-40 \mathrm{~m}$ & $1.7-43 \mathrm{~m}$ & $1-100 \mathrm{~m}$ \\
\hline Repeat cycle & 11 days & 6 days & 14 days \\
\hline
\end{tabular}

Table 1. Spaceborne SAR images commonly used

Our monitoring system considers Sentinel-1 images as priority due to its versatile suitability. Five advantages are described in the following. First, the use of $\mathrm{C}$ band leads to a compromise result between $\mathrm{X}$ and $\mathrm{L}$ bands. $\mathrm{X}$ band is able to measure small deformation while the results suffer from coherence lose (accuracy degradation) especially in vegetation areas. In contrast, $\mathrm{L}$ band is more robust against such a coherence loss; however, small deformation might not be detected. Mostly, $\mathrm{C}$ band satisfies the requirements for monitoring missions. Second, a standard image package contains a large area thanks to TOPSAR acquisition mode. Such a large coverage enables synchronous monitoring of multiple mines and areas of interest. Third, the meter-level resolution is sufficient to monitor mining impact. In most cases, sub-meter level spatial resolution is unnecessary. Fourth, the shortest repeat cycle of 6 days empowers weekly monitoring. Last but not least, Sentinel-1 images are free of charge for both scientific and commercial purposes.

If needed, we will turn to $\mathrm{X}$ or $\mathrm{L}$ bands in some cases. For example, high-resolution TerraSAR-X images could bring more measurement points and more accurate results. PALSAR-2 images are preferred if the monitoring areas are covered with dense vegetation.

\subsection{Interferometry techniques}

DInSAR using only two SAR images computes surface movement commonly up to centimetre level. This method is fast and mainly used to evaluate large-scale surface movement during a limited period, e.g., seismic crustal displacement or slope sliding. There are two main limitations. First, atmospheric noise cannot be effectively removed. Second, noise caused by temporal decorrelation between two images often degrades resultant quality. These two drawbacks are overcome in PSI and SBAS by using multi-temporal SAR images.

PSI and SBAS possess their own pros and cons and are adapted along with adequate strategies for scene monitoring. PSI detects and analyses coherent measurement points from a SAR image sequence. These measurement points are characterized by stable, strong, and coherent signals reflected from a ground patch like a piece of a tailing dam. They are less affected by temporal decorrelation and contain mostly meaningful information. The average velocity (up to sub-millimetre/year accuracy) and timeseries displacements are then evaluated for each point. Atmospheric noise are filtered out by using a spatiotemporal filtering to the image series. However, measurement points are barely found from low- and moderate-coherence areas such as vegetation cover. Similar to PSI, SBAS also adopts multitemporal SAR images to monitor ground deformation. The difference is that SBAS is capable of providing meaningful 
results over low- and moderate-coherence areas; nevertheless, the deformation accuracy is lower (up to millimetre/year) compared with PSI.

In addition to small deformation, ground changes like expansion of an open mining pit can be identified by change detection of SAR images (Preiss and Stacy, 2006). In principle, this methodology looks for singular amplitude or coherence changes as signs of change events. For example, shrinkage and expansion of tailing waste cause coherence variation. We also include a novel PSI-based change detection (Yang and Soergel, 2018), which delivers point-based change information regarding space and time.

\subsection{Refinement}

After interferometry processing, deformation estimates still contain unwanted components (atmospheric delay, thermal noise, temperature, soil moisture, rainfall, noise, etc.), which lead to misinterpretation. For instance, variation of soil moisture could cause heave and subsidence in fields (Zwieback et al., 2017). Such up-and-down movement is then mixed with mininginduced deformation of interest. We apply a temporal filtering to each point to refine its time-series displacements. The meaningful movements remain assuming they dominate over other components. The filtering should be adapted for different land covers. For instance, compared with urban areas, we expect a stronger filtering to fields, which normally causes serious noise.

We also involve statistics in this step to come up with reasonable interpretation. There are two important aspects. First, one point might undergo up-and-down ground movement. Therefore, we separate rising and sinking displacements and calculate their selfcontained statistics for each point, i.e., mean, maximum, minimum, standard deviation, maximum difference, acceleration, and so on. Both rising and sinking velocities are thus presented instead of only an average velocity, which is commonly seen all over. Second, interferometry results are relative and usually based on a reference point. We further correct possible systematic biases by a data-driven way. The time-series displacements of all points are assumed to comply with Normal distribution centring on 0 except those large movements. The biases can then be compensated according to the shift away from 0 .

Furthermore, ground truth data are also considered to refine our results. Commonly, ground control points, e.g., GNSS and levelling benchmark, are used to calibrate deformation estimates. We also want to utilize climate data such as temperature, rainfall, and soil moisture for this purpose. They can be acquired from, for example, Deutscher Wetterdienst (DWD) free of charge. The movements due to these natural factors should be removed to bring the real movements of interest. The main challenge is how to convert such complementary data to be comparable to interferometry results. We must also develop proper inversion algorithm.

\subsection{Analysis}

We must interpret the information delivered to customers along with quality description. For SAR community, coherence is usually used to evaluate precision of interferometry results. The higher a coherence of a point is, the higher precision of its result is expected. However, it is just a number between 0 and 1 and doesn't make any sense to the public. Our system converts wavelength and coherence to a metric form to evaluate the precision of a movement estimate as

$$
\sigma_{\mathrm{M}}=\frac{\lambda}{4 \pi} \sqrt{\frac{1-\gamma^{2}}{2 \gamma^{2}}}
$$

where $\lambda$ and $\gamma$ are wavelength and coherence, respectively.

For each monitoring instance, we would double-check the consistency by comparing the results derived from different methods (e.g., PSI and SBAS) and data set (e.g., ascending and descending images). Inconsistent results cause suspicion of errors, e.g., a point shows sign of heave in an ascending result but manifests subsidence in a descending example. In this case, we must go thoroughly the results to identify the errors.

Behaviours of points are characterized by their essences. For instance, coherences evaluated from tailing areas are expected to be lower than those from dam surfaces. Given a reverse situation, the coherences might be either under- or overestimated, leading to false information. Our system considers point labels in postprocessing to improve the accuracy.

Except the data-driven ways mentioned above, we will employ ground truth to check and improve interferometry results. The current plan includes data derived from GNSS, Levelling, and corner reflectors. Their data formats, geographic locations, and temporal sampling are converted to be comparable to interferometry results. Fixed points can be used as reference or check points. If check points are moving, we suggest to always compare the movements along line of sight (LOS), i.e., look of SAR sensors. The reason is to keep the most accurate results from interferometry and avoid ambiguity in moving orientation as far as possible.

\section{TEST}

Our initial test site belongs to an active open-pit mining area El Soldado in Chile, operated by Anglo American. The task is to monitor dam El Torito (Figure 2), which holds the tailings released after orc refinement. The dam is currently instrumented and surveilled with piezometers and settlement plates. The whole area is mapped by drones twice per month. In addition, survey campaigns are regularly implemented to measure dam density, infiltration flow, and so on.

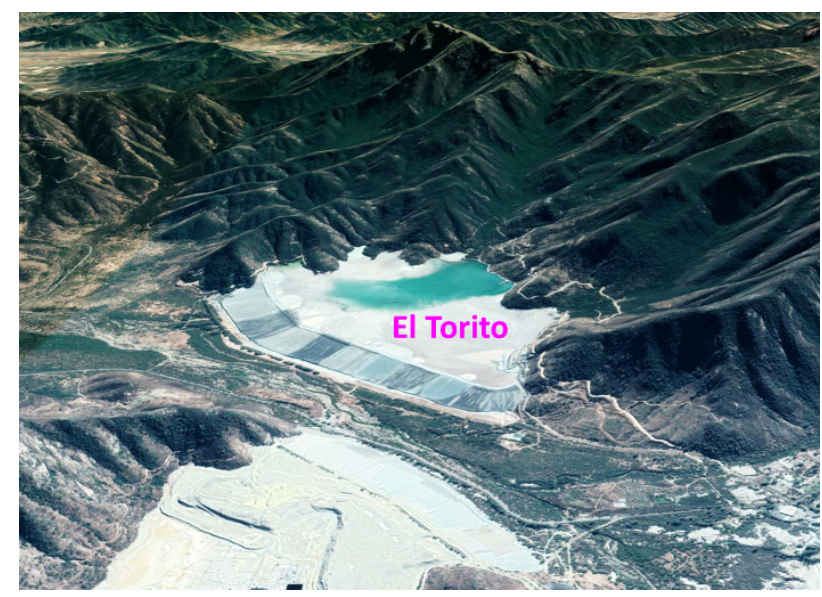

Figure 2. Tailing dam El Torito in Chile

We are working to test and add satellite-based interferometry to monitor dam stability, including surface deformation over the surrounding region. The system is able to provide regular measurement results covering the whole mining area. In addition, we are planning to install 10 GNSS devises and 5 corner 
reflectors on the dam. The corner reflectors will be placed and integrated at the same positions of GNSS devices. The data delivered from these instruments will be used to calibrate, check, and assess the interferometry results.

The SBAS result displays a point-based deformation map (Figure 3 ). We have discussed this initial result with our technical and local partners during the site visit in October, 2018. The result was examined and regarded as sensible although ground truth data are not yet used for double check. The dam is subject to deformation caused by compaction. The maximum movement occurs on top of the dam. One of the reasons could be that new tailings are released and accumulated from many vents framed on the top. In addition, there are routinely working activities such as maintenance, survey, transport, and so on. In contrast, the bottom sections are fixed as the mud-like materials should have been compacted to the utmost limit without interspace. Here we demonstrate the feasibility of providing long-term and largescale monitoring results in a cost-effective way. All of the Sentinel-1 images are costless and the SBAS processing took us merely hours. Later we will involve ground truth data in our tests and analysis. These data will be obtained from in situ surveys, GNSS, corner reflectors, and so on.

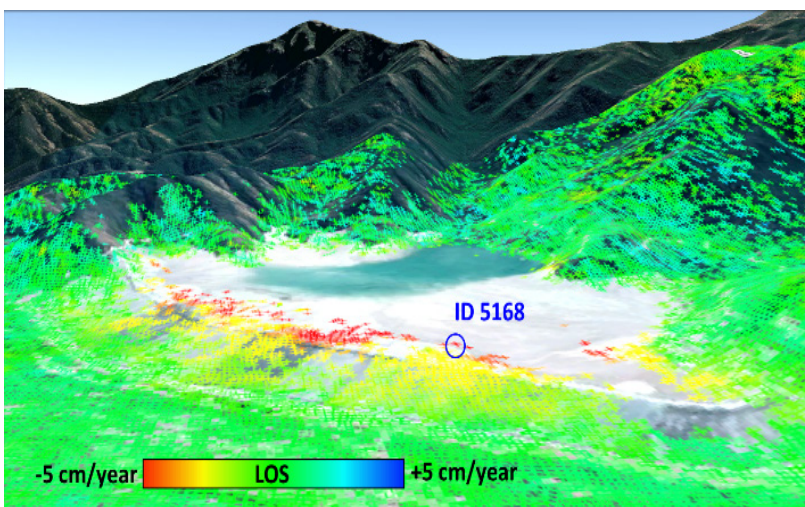

Figure 3. SBAS deformation map produced from Sentinel-1 images taken in 2017. Positive and negative LOS velocities imply rising and sinking movements, respectively. Point ID 5168 used for further discussion. Background, Google Earth image.

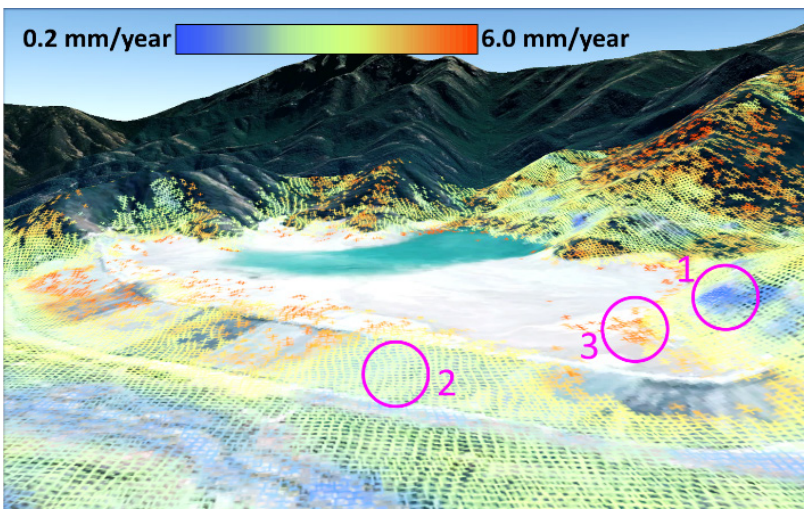

Figure 4. SBAS deformation precision map (mm/year). Areas 1, 2, 3: facility, dam, tailing. Background, Google Earth image.

The deformation precision map (Figure 4) assesses our SBAS result. In principle, precisions are correlated with coherences of different land types. The most precise points belong to the highcoherence facilities (area 1). The dam slope (area 2) shows rather moderate coherence, leading to a second precision level. The precision on tailing always tends to be low as they are usually subject to large temporal decorrelation. In this example, the precision degree conforms to a general sense that we expect.

Each point possesses a set of attributes. For instance, point ID 5168 contains coordinate: $\left(-32.642^{\circ} \mathrm{N},-71.164^{\circ} \mathrm{E}\right)$, velocity: $4.7 \mathrm{~cm} /$ year, velocity precision: $0.5 \mathrm{~cm} /$ year, height: $376.1 \mathrm{~m}$, height precision: $5.0 \mathrm{~m}$, etc. The deformation $(-4.7 \mathrm{~cm} /$ year $)$ is noticeable with high precision $(0.5 \mathrm{~cm} /$ year $)$. More attributes will be added if necessary. For example, point label indicates dam, tailing, facility, bare ground, or vegetation. A risk index quantifies how likely a local dam damage might be caused due to movement. We also compare the time-series displacements derived by PSI and SBAS (Figure 5). They match consistently and show the same subsidence trend. This consistency implies that both PSI and SBAS results convey the real instance.

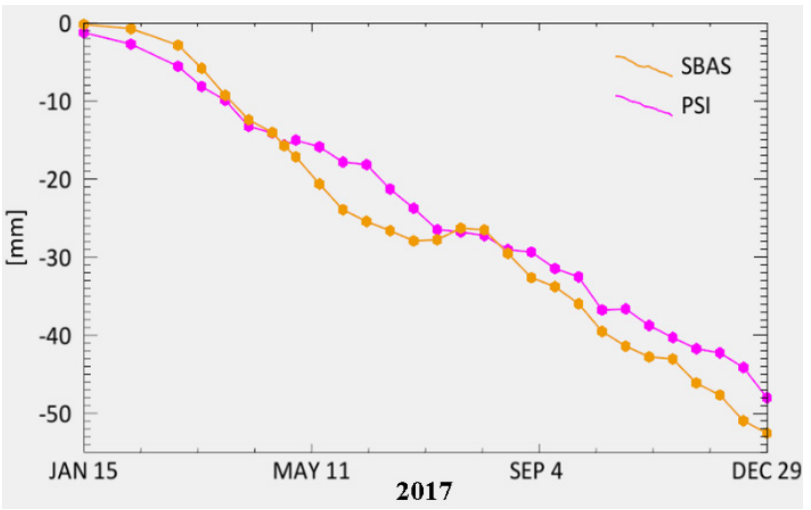

Figure 5. Displacement sequence of point ID 5168.

In the near future, we will use ground truth data to validate and improve our interferometry results. We are planning to install 10 GNSS devices and 5 corner reflectors across the dam (Figure 6). A corner reflector will be integrated with one GNSS devise at the same position. The final decision and campaign are still under discussion. Afterwards, the in situ measurement data will be collected, processed, and transmitted to our storage server.

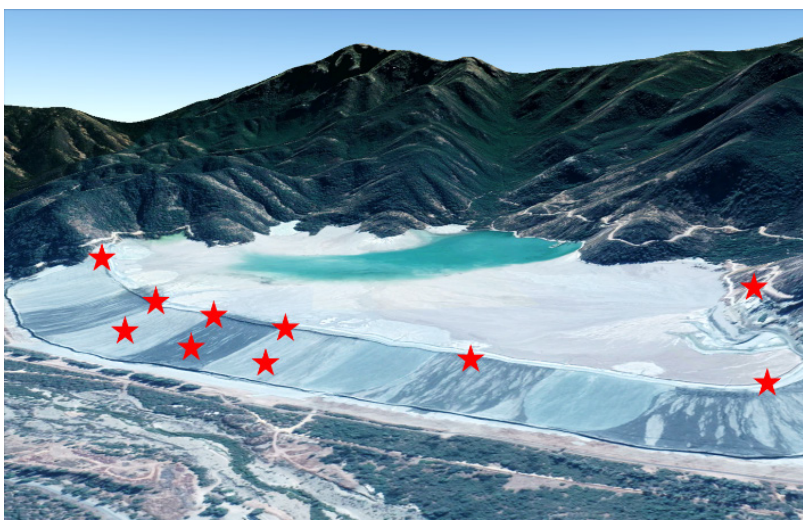

Figure 6. Planned positions (red asterisks) of 10 GNSS devices. 5 corner reflectors will be integrated to five of these positions (under discussion).

\section{REALISTIC PROBLEMS AND SOLUTIONS}

\subsection{Mixture of movement components}

Interferometry can be regarded as an opportunistic measurement We don't know where and what we will really measure and obtain. Only after processing, the measurement points are geocoded and projected to a map so that the corresponding targets can be identified, e.g., dam, building, bare ground, etc. We then 
interpret the movements along with their coherences, precisions, statistics, and so on. The most concern is that where these movements come from. Each movement may contain real components of interest, e.g., dam deformation, and unwanted terms caused by temperature, soil moisture (rainfall), noise, errors, and so forth. This mixture leads to misinterpretation of monitoring results. For example, if the aim is to evaluate the subsidence in a residential district, the seasonal displacements due to variation of temperature and soil moisture should be filtered out.

For refinement, a temporal low-pass filtering is applied to displacement series of each point to remove unwanted noise. Our test exemplifies a stable point over a building and a sinking point in field (Figure 7). We suppose the original displacement sequence (blue, Figure 8) of the stable point is mainly subject to temperature and rainfall plus a minority of random noise. Most of the noise is suppressed after low filtering (orange, Figure 8). The resultant curve is positively related to the average temperature and rainfall combined (Figure 9). The higher temperature and rainfall, the more upward the target is moving. The reasons are that warm temperature causes building expansion and heavy rainfall possibly swells the underground soil. After a stronger filtering, the climate effect has been almost eliminated (red, Figure 8). As a result, we do not see significant movements and therefore infer that the building is stable. Without this filtering step, people would think the building underwent longterm and up-and-down vibration.

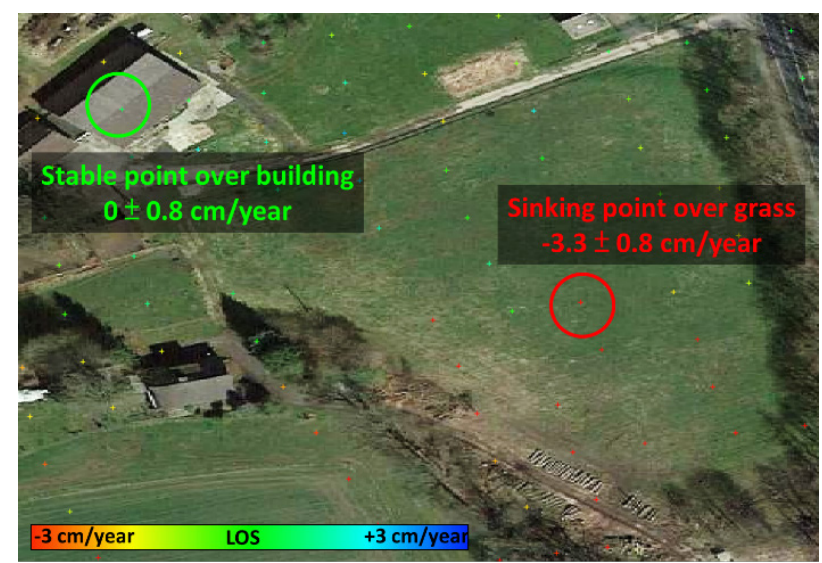

Figure 7. Examples of stable and sinking points in SBAS deformation map (Hattingen, Germany, 2015).

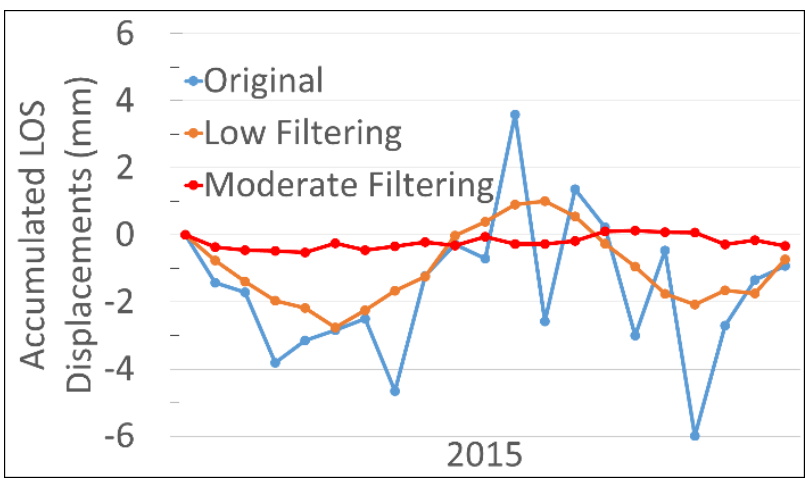

Figure 8. Time-series displacements of stable point (Figure 7). Blue, original; Orange, low filtering; Red, moderate filtering.

We suppose that the time-series displacements of the sinking point (blue, Figure 10) are originally attributed to significant subsidence, climate effect, and random noise. Unlike the instance of the stable point (orange, Figure 8), the processed curve (orange, Figure 10) does not clearly manifest the components of temperature and rainfall after low filtering. This is because the subsidence dominates over other effects. We then applied a high filtering, which resulted in a pure subsidence curve (red, Figure 10). However, we also notice that the original curve might indicate a sudden subsidence. This possibility can be also seen in the example of the stable point (Figure 8). To avoid missed detection, we should develop a multi-scale analysis on displacement sequences.

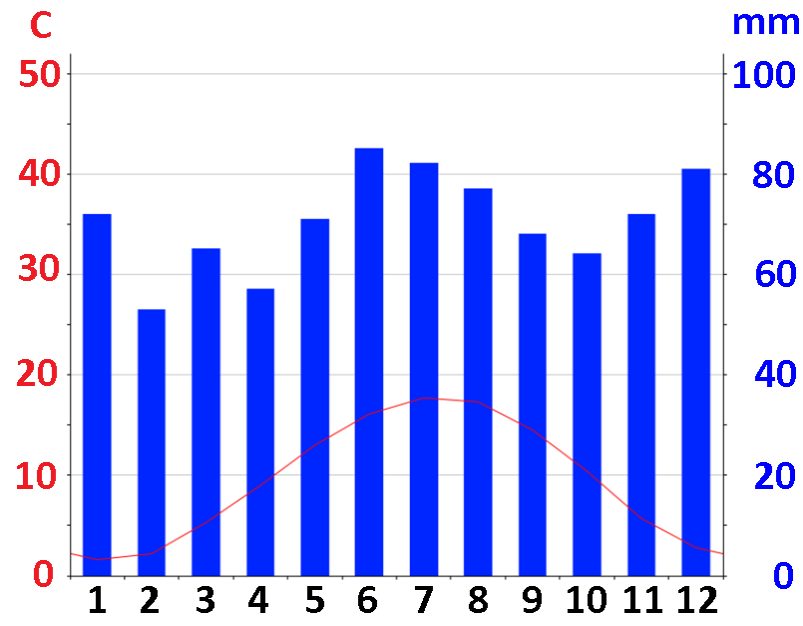

Figure 9. Average yearly temperature (red) and rainfall (blue) (Hattingen, Germany, 1982 - 2012) from CLIMATE-

DATA.ORG

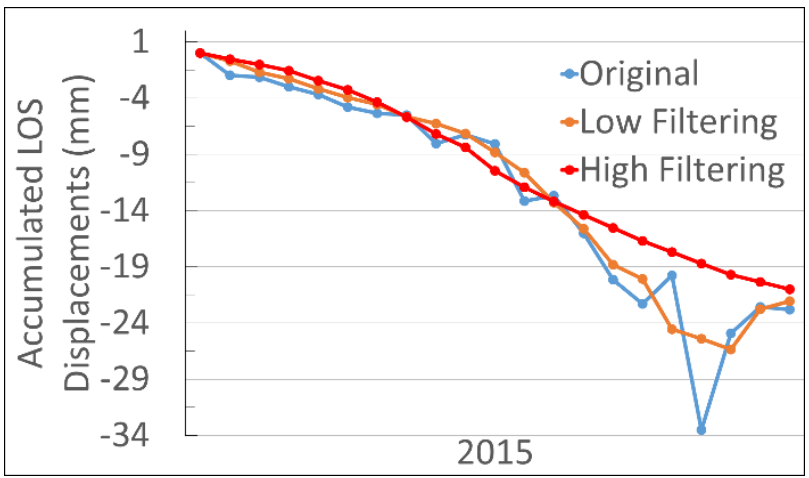

Figure 10. Time-series displacements of sinking point (Figure 7). Blue, original; Orange, low filtering; Red, high filtering.

We have found a promising way of filtering to extract real movements of interest. The filtering strength can be adjusted based on ground truth data such as ground control points and upto-date climate data (Deutscher Wetterdienst). Alternatively, we would derive movement components directly from climate data and remove them in final result.

\subsection{How to detect risky points?}

There are often tremendous points of various deformation behaviours in an interferometry result. Which ones are more crucial for the task of interest like safety? The definitions must be clarified in the very beginning. For instance, a sinking point is treated as a risk if its movement behaviour diverges greatly from others within a neighbour. This divergence could cause structural damages at the position. Here we demonstrate how to detect a risky point by using an area-based heterogeneity test. The water pipe at point 1 was confirmed to break in early 2016 (Figure 11). The point was remarkably sinking $(-29 \mathrm{~mm} /$ year $)$ in 2015 , which 
shows a sign of a potential risk. The average and STD of velocities within neighbour 1 are -6 and $7 \mathrm{~mm} /$ year, respectively. The velocity of the sinking point is far greater than the average and even than triple STD. We also plot and compare the displacement sequences of the sinking point and average (Figure 12). These two sequences greatly diverge from each other. The divergence is quantified by Euclidean distance: $36 \mathrm{~mm}$, Manhattan distance: $125 \mathrm{~mm}$, and Chebyshev distance: $16 \mathrm{~mm}$. Finally, this sinking point is labelled as a risky point, which could be the cause of the break event in 2016.

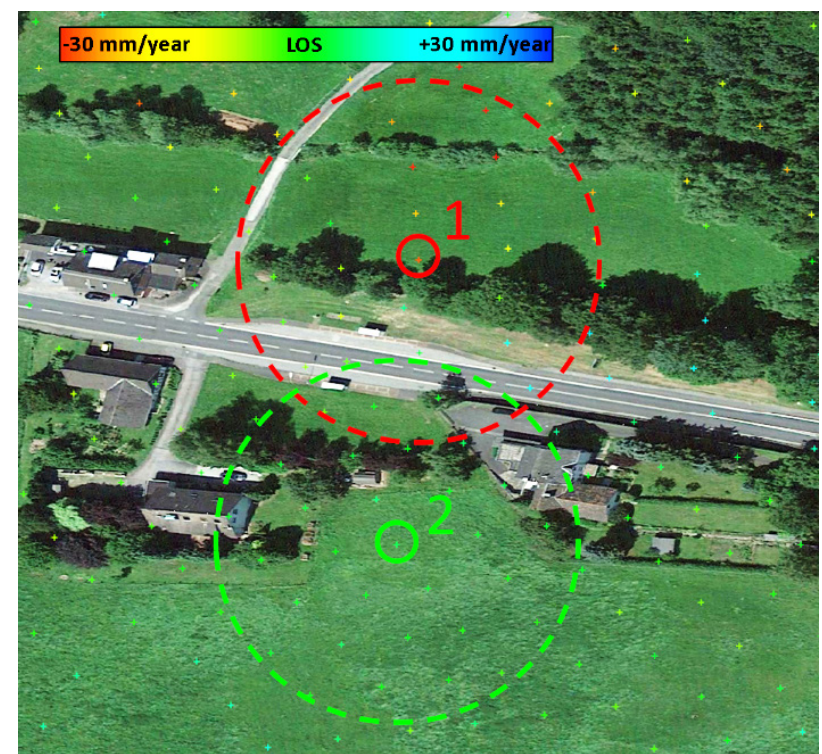

Figure 11. SBAS deformation map (Hattingen, Germany, 2015). Sinking point $1(-29 \mathrm{~mm} /$ year $)$ at venue of water pipe break. Neighbour 1 of diameter $50 \mathrm{~m}$ : average, $-6 \mathrm{~mm} /$ year; STD, 7 $\mathrm{mm} /$ year. Stable point $2(-1 \mathrm{~mm} /$ year $)$ at grass. Neighbour 2 of diameter $50 \mathrm{~m}$ : average, $-2 \mathrm{~mm} /$ year; STD, $3 \mathrm{~mm} /$ year.

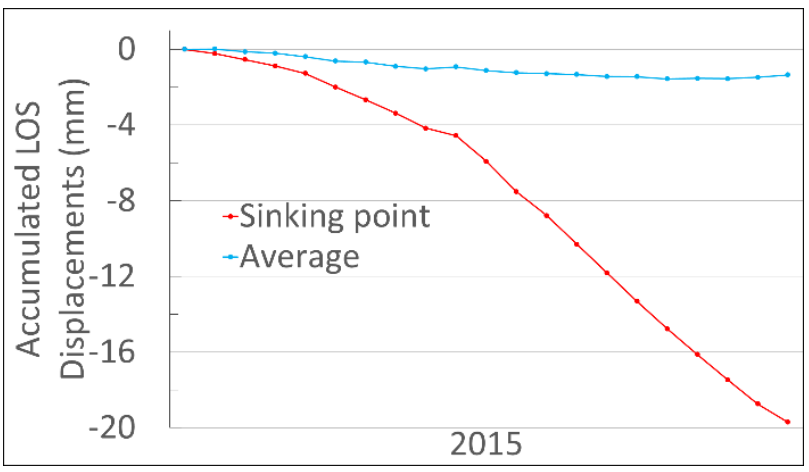

Figure 12. Displacement sequences of sinking point (red) and average (blue) (Figure 11). Similarity between two sequences: Euclidean distance, $36.4 \mathrm{~mm}$; Manhattan distance, $124.7 \mathrm{~mm}$; Chebyshev distance, $16.1 \mathrm{~mm}$.

We also analysis stable point 2 in a similar way (Figure 11). The negligible velocity does not show a meaningful difference from others after comparing with the average and STD. The displacement sequences of the stable point and average match very well given Euclidean distance: $3 \mathrm{~mm}$, Manhattan distance: $12 \mathrm{~mm}$, and Chebyshev distance: $1 \mathrm{~mm}$ (Figure 13). The target point is then regarded as stable without warning.

This test shows risky points can be identified automatically or at least quantified by certain statistics. The relevant parameters should be determined based on a priori knowledge and ground truth. For instance, how large a similarity distance indicates a risky point? We will continue this topic with our project partners and local experts.

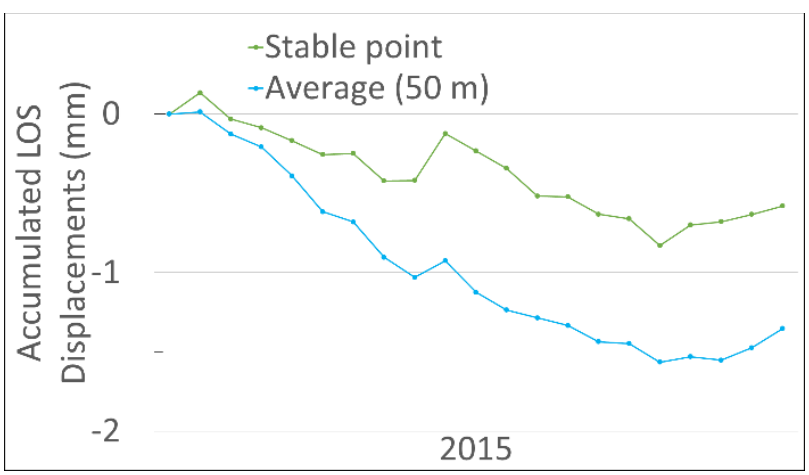

Figure 13. Displacement sequences of stable point (green) and average (blue) (Figure 11). Similarity between two sequences:

Euclidean distance, $3 \mathrm{~mm}$; Manhattan distance, $12 \mathrm{~mm}$; Chebyshev distance, $1 \mathrm{~mm}$.

\subsection{Bottom line}

The problems and solutions mentioned above concern general interferometry applications not just for mining. We are working to improve the proposed methods to be more elegant, precise, and automatic. For this purpose, we will also involve ground truth data in analysis. Another plan is to refine certain point attributes for following analysis, i.e., velocity, displacement sequence, coherence, precision, etc. More possibilities will be discussed in STINGS.

\section{CONCLUSIONS}

We have proposed the initial prototype of our monitoring system developed under STINGS project (https://eitrawmaterials.eu/pro ject/stings/). Using spaceborne SAR images, the system delivers point-based deformation and change detection maps. The results can be converted to other formats or thematic maps. This paper focuses on monitoring of dams and tailing in mines by interferometry techniques including DInSAR, PSI, and SBAS. The system will be later adapted for other fields. The final goal is to launch an interactive GIS-based platform as an early warning system to the public. For example, the authority will be informed if there is a sign of structural damage on a dam, which might cause a catastrophic disaster.

Our test result shows a SBAS deformation map over dam El Torito in Chile. The result was examined and regarded as sensible after discussion with our technical and local partners during a site visit. We also demonstrate how to interpret the deformation map with proper data-driven statistics and strategies. Each point is assessed by its own precision and label. For example, we have seen a rational fact that the points on the high-coherence facilities are more precise than those on the low-coherence tailing with respect to deformation velocities. The displacement sequences of a point in PSI and SBAS results are also compared to validate consistency. Currently, we are planning to install 10 GNSS devices and 5 corner reflectors across the dam. The data from these devices will be used to validate and improve our approaches.

We also discuss and demonstrate the real problems and solutions commonly seen in practice. First, real movements of interest like ground subsidence are often mixed with other unwanted components caused by, e.g., temperature, rainfall, and noise. A 
temporal filtering is designed to extract real movements while other items are removed. Second, we propose an area-based heterogeneity test to detect risky points, whose movement behaviours greatly diverge from the neighbours. We will continue to improve the current methods to be more elegant, precise, and automatic. We are also planning to refine certain point attributes for following analysis, i.e., velocity, displacement sequence, coherence, precision, etc. More possibilities will be discussed later in STINGS.

\section{ACKNOWLEDGEMENT}

We want to appreciate many supports in our STINGS project funded by EIT (European Institute of Innovation and Technology) RawMaterials. The Sentinel-1 images are provided by Copernicus - European Union's Earth Observation Programme. Anglo American kindly approves our request and access to mine El Soldado as our test site. Many helps also come from our partners: DMT GmbH, Geological Survey of Slovenia, LTU Business AB, Ovidius University of Constanta, Stuttgart University, Technische Universität Bergakademie Freiberg, and niversidad Pontificia Católica de Valparaiso.

\section{REFERENCES}

Berardino, P., Fornaro, G., Lanari, R. and Sansosti, E., 2002. A new algorithm for surface deformation monitoring based on small baseline differential SAR interferogram. IEEE Transactions on Geoscience and Remote Sensing, 40(11), 23752382 .

Crosetto, M., Monserrat, O., Cuevas-González, M., Devanthéry, N. and Crippa, B., 2016. Persistent scatterer interferometry: a review. ISPRS Journal of Photogrammetry and Remote Sensing, $115,78-89$.

Ferretti, A., Prati, C. and Rocca, F., 2000. Nonlinear subsidence rate estimation using permanent scatterers in differential SAR interferometry. IEEE Transactions on Geoscience and Remote Sensing, 38(5), 2202-2212.

Ferretti, A., Prati, C. and Rocca, F., 2001. Permanent scatterers in SAR interferometry. IEEE Transactions on Geoscience and Remote Sensing, 39(1), 8-20.

Ferretti, A., Fumagalli, A., Novali, A., Prati, C., Rocca, F. and Rucci, A., 2011. A new algorithm for processing interferometric data-stacks: SqueeSAR. IEEE Transactions on Geoscience and Remote Sensing, 49(9), 3460-3470.

Hooper, A., Zebker, H., Segall, P. and Kampes, B., 2004. A new method for measuring deformation on volcanoes and other natural terrains using InSAR persistent scatterers. Geophysical Research Letters, 31(23), 1-5.

Kampes, M., 2006. Radar Interferometry: Persistent Scatterer Technique. Springer.

Lanari, R., Casu, F., Manzo, M., Zeni, G., Berardino, P., Manunta, M. and Pepe, A., 2007. An overview of the small baseline subset algorithm: a DInSAR technique for surface deformation analysis. Pure and Applied Geophysics, 164(4), 637-661.

Preiss, M. and Stacy, N.J.S., 2006. Coherent Change Detection: Theoretical Description and Experimental Results. Defence Science and Technology Organisation.
Yang, C.-H. and Soergel, U., 2018. Adaptive 4D PSI-based change detection. ISPRS Ann. Photogramm. Remote Sens. Spatial Inf. Sci., IV-3, 245-250, https://doi.org/10.5194/isprsannals-IV-3-245-2018.

Zwieback, S., Hensley, S., and Hajnsek, I., 2017. Soil moisture estimation using differential radar interferometry: toward separating soil moisture and displacements. IEEE Transactions on Geoscience and Remote Sensing, 55(9), 5069-5083. 